\title{
A multicentre evaluation of a questionnaire to assess ability in the diagnosis of leprosy
}

\author{
W C S SMITH \\ Cardiovascular Epidemiology Unit, Ninewells Hospital and Medical \\ School, Dundee DDI 9SY, UK
}

Accepted for publication 11 September 1986

\begin{abstract}
Summary Case finding in leprosy control programmes is dependent on the ability of field staff in the diagnosis of leprosy. However assessment of this ability in field circumstances is difficult and time consuming. In this study 20 case histories in the form of a questionnaire is evaluated as a tool to assess ability to interpret the signs and symptoms of leprosy. The study included field workers of different grades and differing experience from 6 centres in India. The validity of this method is shown by the better performance by the higher grades of staff and the correlation of performance with experience. The use of case histories in this way is a useful educational tool; it can also be used to identify individual members with major difficulties in the diagnosis of leprosy and to identify particular cases which present more general diagnostic problems.
\end{abstract}

\section{Introduction}

In most leprosy control programmes case finding is undertaken by paramedical or basic health workers by population screening. The diagnosis of leprosy in the field is based on illiciting clinical signs and symptoms; laboratory investigations such as skin-smear examination or histopathology play a confirmatory role at a later stage if at all. The clinical diagnosis made by the paramedical staff is then usually confirmed by a more senior supervisor or medical officer before active chemotherapy is initiated.

This approach to case detection uses standard screening methodology and it is thus appropriate to apply screening criteria ${ }^{1}$ to assess the detection of leprosy in control programmes. The paramedical staff perform the standard screening test while the senior staff act as the standard and valid diagnostic test. Thus the screening examination can be assessed for specificity, sensitivity and overall agreement. The re-examination by senior staff of cases positive by the screening examination gives an estimate of the false positives. However to estimate the numbers of false negatives it requires the senior staff to repeat the population 
screening at a similar point in time but independent from the paramedical workers examination, this has been undertaken in special surveys and it has been suggested that $5-10 \%$ of the population be regularly resurveyed. ${ }^{2}$ Such reexamination is costly and programme directors may not be convinced of their necessity.

The whole procedure of population screening is complex, it is dependent on community co-operation, a high level of coverage, the ability to conduct a full examination in a good light under difficult circumstances, and the expertise to interpret the findings in making a diagnostic decision. It is, however, possible to identify parts of the process and assess these rapidly and inexpensively in isolation. Such methods can detect problems and be part of quality assurance in leprosy control work as well as contributing to the ongoing in-service training of field staff in leprosy control programmes.

In this study the efficacy of a simple questionnaire to assess the ability of leprosy control programme staff to interpret clinical findings in the diagnosis of leprosy is evaluated. The validity of the tool is assessed by examining the results from different programmes with staff of different grades and levels of experience.

\section{Methods}

Twenty case histories of typical problems encountered in case detection in a leprosy control programme were prepared. These twenty were chosen to represent common problems and are therefore not necessarily typical of all suspected cases. The 20 case histories are given in the Appendix along with the standard answers used in the analysis. Each member of the field staff reads each history and then writes down his diagnosis as one of three categories, affected, not affected or suspected, as is the standard method in population screening.

Copies of the 20 case histories were completed independently by the field staff of 6 different leprosy control programmes throughout India and by paramedical workers undergoing initial training. A set of 'correct' answers were prepared and each worker's responses were compared with this standard. The agreement between the field worker and the standard is expressed as a percentage of all cases. The number of cases considered suspects is presented as a percentage of all the cases. Cases considered affected by the field worker and not affected by the standard are described as false positives and are expressed as a percentage of all diagnosed not affected by the standard. Similarly false negatives were those considered unaffected by the field worker but affected by the standard. The performance of each worker was thus assessed for overall agreement, the proportion of suspects, false positives and false negatives.

The standard answers used in the analysis have been compared with the majority answer. Difficulty and discrimination indices have also been calculated for each of the 20 cases based on the findings. 
Table 1. Performance of the six centres

\begin{tabular}{cccccc}
\hline & $\begin{array}{c}\text { Number of } \\
\text { Centre }\end{array}$ & $\begin{array}{c}\text { Agreement } \\
(\%)\end{array}$ & $\begin{array}{c}\text { Suspects } \\
(\%)\end{array}$ & $\begin{array}{c}\text { False } \\
\text { negatives }\end{array}$ & $\begin{array}{c}\text { False } \\
\text { positives }\end{array}$ \\
\hline 1 & 14 & 53 & 31 & $8 \cdot 3$ & $11 \cdot 4$ \\
2 & 26 & 48 & 32 & $12 \cdot 2$ & $15 \cdot 4$ \\
3 & 7 & 62 & 27 & $4 \cdot 8$ & $11 \cdot 4$ \\
4 & 11 & 56 & 30 & $4 \cdot 5$ & $5 \cdot 4$ \\
5 & 17 & 55 & 34 & $10 \cdot 8$ & $8 \cdot 2$ \\
6 & 9 & 55 & 36 & $11 \cdot 2$ & $11 \cdot 2$ \\
\hline \multirow{2}{*}{ Total } & 84 & 53 & 31 & $9 \cdot 5$ & $11 \cdot 2$ \\
\hline
\end{tabular}

Table 2. Performance in the 4 grades of staff

\begin{tabular}{lccccc}
\hline \multicolumn{1}{c}{ Grade } & $\begin{array}{c}\text { Number of } \\
\text { staff }\end{array}$ & $\begin{array}{c}\text { Agreement } \\
(\%)\end{array}$ & $\begin{array}{c}\text { Suspects } \\
(\%)\end{array}$ & $\begin{array}{c}\text { False } \\
\text { negatives }\end{array}$ & $\begin{array}{c}\text { False } \\
\text { positives }\end{array}$ \\
\hline Trainees & 21 & 46 & 29 & $11 \cdot 8$ & $18 \cdot 0$ \\
Paramedical workers & 51 & 54 & 32 & $9 \cdot 5$ & $11 \cdot 0$ \\
Non-medical supervisors & 6 & 67 & 26 & 0 & 0 \\
Medical officers & 6 & 62 & 34 & $11 \cdot 2$ & 0 \\
\hline Total & 84 & 53 & 31 & $9 \cdot 5$ & $11 \cdot 2$ \\
\hline
\end{tabular}

\section{Results}

The 20 case histories were completed by 84 field workers from 6 centres including 21 trainee paramedical workers, 51 trained paramedical workers (PMW), 6 nonmedical supervisors (NMS) and 6 medical officers (MO). The results from each centre are shown in Table 1. The pattern in each centre is similar. Centre 2 contained the 21 trainees and thus had the lowest agreement and highest percentage of false negatives and positives. Each centre was a mixture of staff of differing grades and of different experience.

The performance of the 4 grades of staff is shown in Table 2 . The performance of trainee paramedical workers when compared with the standard shows the least agreement and the most false diagnoses. The performance of the supervisors and medical officers is better than that of the paramedical workers. The effect of experience in the field of leprosy on performance is assessed in Table 3. Five participants failed to give details of their experience. There is a general trend of 
Table 3. Performance and years of experience

\begin{tabular}{cccccc}
$\begin{array}{c}\text { Experience } \\
\text { in years }\end{array}$ & $\begin{array}{c}\text { Number of } \\
\text { staff }\end{array}$ & $\begin{array}{c}\text { Agreement } \\
(\%)\end{array}$ & $\begin{array}{c}\text { Suspects } \\
(\%)\end{array}$ & $\begin{array}{c}\text { False } \\
\text { negatives }\end{array}$ & $\begin{array}{c}\text { False } \\
\text { positives }\end{array}$ \\
\hline 0 & 22 & 47 & 32 & $12 \cdot 2$ & $17 \cdot 2$ \\
1 & 7 & 62 & 35 & $7 \cdot 2$ & $5 \cdot 8$ \\
2 & 18 & 52 & 34 & $13 \cdot 0$ & $12 \cdot 2$ \\
3 & 15 & 54 & 31 & $6 \cdot 7$ & $8 \cdot 0$ \\
$>3$ & 17 & 61 & 29 & $6 \cdot 0$ & $3 \cdot 6$ \\
\hline Total & 79 & 54 & 32 & $9 \cdot 5$ & $10 \cdot 4$ \\
\hline
\end{tabular}

( 5 failed to give details of their experience)

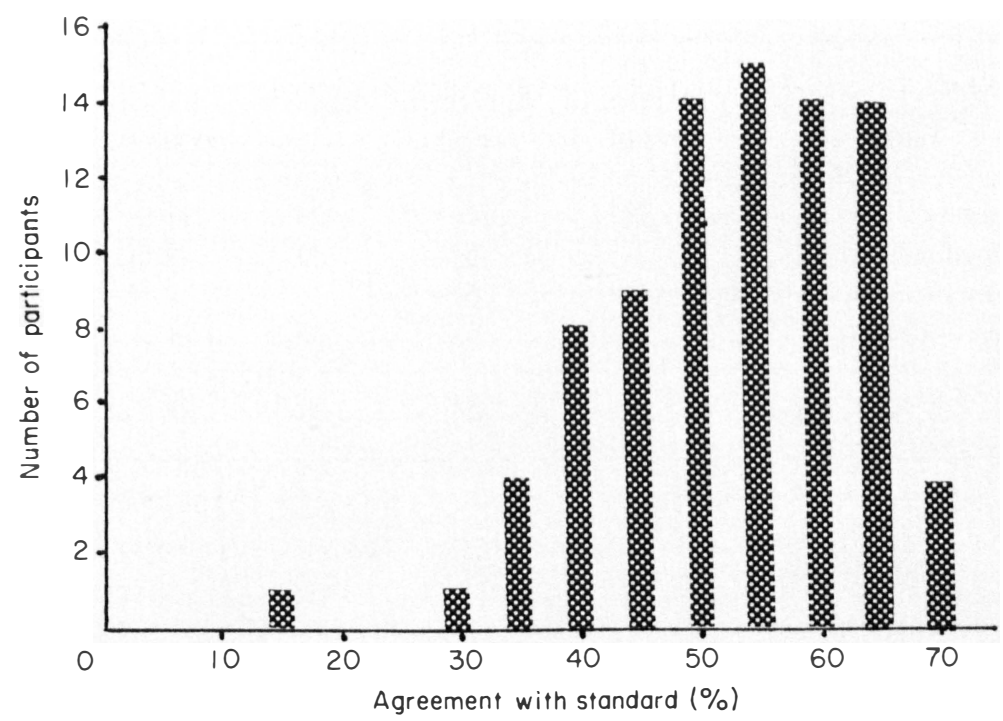

Figure 1. The distribution of the agreement with the standard for the 84 participants.

improved performance with increasing years of experience. The correlation coefficient of experience against agreement $(r=+0 \cdot 4)$ and experience against false positives $(r=-0 \cdot 3)$ are both statistically significant $(p<0 \cdot 05)$.

Each participant's response was assessed for serious deviation from the standard. Serious deviation was considered as any one of the following 6 parameters; failure to detect half the true positives or half the true negatives, more than 1 false negative or false positive, agreement in less than 8 of the 20 cases, and 10 or more suspects. Serious deviations from the standard were detected in 29 
individuals, 14 of whom were trainees. Ten of the remaining 15 had one deviation, 2 had two deviations, 2 had three deviations and one had 5 deviations.

The distribution of the agreement with the standard for each of the 84 participants approximates to normal (Figure 1). From this distribution it is possible to identify the characteristics of those with high ( $65 \%$ or more) and low (40\% or less) scores. The 14 with low scores included 9 trainee PMWs and 5 trained PMWs (2.5 years average experience). The 18 with high score included 1 trainee PMW, 7 trained PMWs (5.5 years average experience), all 6 of the NMSs and 4 out of the 6 MOs.

The standard answers were compared with the majority answer for each of the 20 questions. There was agreement in all but 3 questions $(4,10$ and 19) where in each case the standard was 'suspect' but the majority went for 'unaffected'. A difficulty index was calculated for each question based on the lack of agreement between the 84 participants; where less than $50 \%$ agreed on the answer it was classed difficult and where more than $80 \%$ agreed on the answer it was classed as easy. Using this method, 6 questions were assessed as difficult $(1,8,9,12,14$ and $20)$ and 3 were classed as easy (3, 6 and 15). Each question was then assessed by a discrimination index based on the proportion of participants giving either false positive or false negative diagnoses. Using this index there were 3 questions where more than $10 \%$ gave false positive answers $(5,7$ and 20$)$ and 2 where more than $10 \%$ gave false negative answers (13 and 18$)$.

\section{Discussion}

The analyses of the results of this multicentre evaluation of 20 case histories as a method of assessing ability in the diagnosis of leprosy show a consistency in the responses. The validity of this method of assessment is confirmed by the better performance, across a number of parameters, of the higher grades of staff and those with more experience. This pattern exists for the overall agreement with the standard responses as well as the number of false negatives and false positives. Further, as would be expected in any valid assessment, those in training had the poorest results for all the measures.

The group with the lowest scores includes, along with the trainees, 5 trained PMWs who have low scores and a number of serious deviations from the standard. This ability to identify field staff in service who have problems in the diagnosis of leprosy is an important feature of this case history method. The responses to the questions have also been used to identify problem areas. Early lesions in children seem to present a common difficulty; variation in the response to such cases would lead to apparent differences in the leprosy prevalence amongst children in different regions. The discrimination index is useful in identifying important problems, such as case history No. 13, which the majority diagnosed as leprosy but more than 10\% passed over as unaffected; and those with any skin such as case No. 5 where more than $10 \%$ diagnosed as leprosy. 
The need for operational research in leprosy control programmes has been well recognized for many years. ${ }^{3.4}$ However there are only a few reports of such work being undertaken. ${ }^{5.6}$ Perhaps the main reasons for this gap in field work is that such work is extremely laborious and directors of field work are apprehensive about getting into this area in fear of what may be uncovered. This study suggests a simple and inexpensive method of approaching the problem by picking out one particular aspect of leprosy control work and assessing it in isolation. A good performance with this method does not imply that there are no problems but a poor performance does signify that serious problems do exist which deserve further examination.

These case histories can also be used as a method of in-service training by stimulating discussion between staff in these difficult areas. In-service training or any teaching programme could also be assessed by repeating the case histories on the same group at a later stage. New series of case histories could also be compiled and used in a similar way. Use of the case histories may also help to convince programme directors of the need for regular quality assessment in the diagnosis of leprosy in control programmes. ${ }^{2}$

\section{Acknowledgments}

I would like to thank the staff from the 6 centres who willingly helped in this evaluation.

\section{References}

1 Hart CR. Screening in general practice. Churchill Livingstone. Edinburgh; 1977: p. 12.

2 Kumar A, Durai V, Sivaprasad N, Sirumban P. Diagnostic efficiency of paramedical workers in leprosy. Lepr Rev, 1985; 56: 309-14.

3 Noordeen SK. The need for operational studies in leprosy control. Le pr India, 1977; 49: 6.

4 Meade TW. Growing points in leprosy research-epidemiology. Lepr Rev, 1974; $45: 15$.

5 Neelan PN, Noordeen SK, Desikan KV, Ramu G, Prabhu KPM, Christian M. Inter-observer variations in diagnosis and classification of early lesions of leprosy. XI Biennial Conference of the Indian Association of Leprologists, 1979.

- Kumar A, Durai V, Sirumban P. Diagnostic efficiency of paramedical workers involved in leprosy case detection programme. Ind J Lepr, 1984; 56: 626-32.

\section{Appendix}

\section{Questionnaire-Leprosy case detection}

Read the following 20 case descriptions as if they were people you had met in the 
course of survey work and mark them as either Not Affected (N), Affected (A), or Suspect (S). Do not discuss your answers with others until you have completed the questions. No smears or other tests are available.

Letters in circles represent the standard responses used in the analyses.

A 7-year-old boy has a few pale patches on his face with no anaesthesia. His father has lepromatous leprosy.

2 A 4-year-old boy has two pale patches on his buttocks but he will not co-operate and so sensation is not tested.

3 A 32-year-old woman with slight loss of eyebrows. Her face, back and limbs show redness and infiltration.

4 A 7-year-old boy has a few pale patches on his face with no anaesthesia. He has been kept a suspected case for 3 years but there is no improvement.

5 A 24-year-old woman has several raised patches with a white, scaly surface. There is no anaesthesia.

6 A 19-year-old boy has a single anaesthetic patch on his left hand.

7 A 70-year-old man has only loss of eyebrows. No other signs.

8 A 7-year-old girl has a few pale patches on her face with no sensory change.

9 A 14-year-old boy has a patch on his chest which he says is a birthmark but on testing there is sensory change.

10 A 29 -year-old man has a left drop foot but has no patches or loss of sensation.

11 An 8-year-old girl has two patches on her face with no sensory change. She has been a suspect for 1 year but now the patches are more well defined.

12 A 7-year-old boy has a few patches on his face. There is no sensory change.

13 A 27 -year-old man has thickening of both ulnar and lateral popliteal nerves but no patches or sensory change.

14 A 14-year-old boy has a single well-defined patch on his back with no sensory change.

15 A 50-year-old man has had very white patches on his legs for about two years.

16 A 27-year-old woman has numerous patches on her body and loss of sensation on her feet and hands.

17 A 7-year-old boy has a few pale patches on his face. There is no loss of sensation. His father has tuberculoid leprosy.

18 A 5-year-old boy has a few pale patches on his buttock. Sensation is not tested. His mother has tuberculoid leprosy.

19 A 30-year-old woman has many itchy patches with loss of sensation over these patches. 


\section{$148 \quad W C S$ Smith}

20 A 70-year-old woman has tingling in her hands and feet. There are no patches or loss of sensation.

Staff Grade

Experience in Leprosy .................. years 